\title{
Effect of Eugenol on Streptococcus mutans Adhesion on NiTi Orthodontic Wires: In Vitro and In Vivo Conditions
}

\author{
Nassiba Fatene ${ }^{1} \quad$ Khadija Mounaji $^{1} \quad$ Abdelaziz Soukri $^{1}$ \\ ${ }^{1}$ Department of Biology, Physiopathology Molecular Genetics and \\ Biotechnologies Laboratory, Faculty of Sciences Ain Chock, Center of \\ Health \& Biotechnology, Hassan II University, Casablanca, Morocco \\ Address for correspondence Nassiba Fatene, PhD, Lotissement Mona, \\ résidence llyas Appt 2 Val Fleuri, Tangier 90000, Morocco \\ (e-mail: fatenenassiba@gmail.com).
}

Eur J Gen Dent 2021;10:151-157.

\begin{abstract}
Keywords

- Nickel-Titanium

- Streptococcus mutans

- bacterial adhesion

- Eugenol

- Orthodontic wires

Objective The purpose of this study was to compare the antibacterial effect of two types of Eugenol against Streptococcus mutans and then assess the effect of different concentrations of two types of Eugenol on S. mutans adhesion on Nickel-Titanium (NiTi) orthodontic wires in comparison to in vivo wires with fluoride-based hygiene regimen. Materials and Methods Culture of Streptococcus mutans with NiTi orthodontic wires was done. Different media were prepared by adding $100 \%, 50 \%, 25 \%$, and $12.5 \%$ of two origins of Eugenol (one biological obtained by hydrodistillation of Syzygium aromaticum and one chemical already prepared available in drugstores for dental use (Idental, MOROCCO, lot number: UAN/17-211/1). Three sizes of NiTi wires (0.016 inch, $0.016 \times 0.022$ inch, $0.017 \times 0.025$ inch) were retrieved from adult patients undergoing orthodontic treatment after 1 month of setting them up in the mouth. After incubation, colony forming unites were calculated and a SEM analysis was done to the surface of each wire. ANOVA test was done between all groups to find statistical differences and post-hoc $t$-test with Bonferroni analysis was performed to elucidate differences between all groups with $\alpha=0.05$.

Results Eugenol has an anti-bacterial effect against $S$. mutans. The biological Eugenol has greater effect than the chemical one. The same observations were done for antiadherent effect, the biological Eugenol demonstrated the highest anti-adherent effect at all concentrations while the effect of the chemical Eugenol was the lowest.

Conclusions The origin and the extraction mode of Eugenol have a crucial role in its antimicrobial and anti-adherent effect. Eugenol might constitute an alternative to Fluoride because it has an anti-adherent effect, limiting the incidence of white spot lesions.
\end{abstract}

DOI https://doi.org/ $10.1055 / \mathrm{s}-0041-1736372$. ISSN 2278-9626.

\footnotetext{
(c) 2021. European Journal of General Dentistry. All rights reserved. This is an open access article published by Thieme under the terms of the Creative Commons Attribution-NonDerivative-NonCommercial-License, permitting copying and reproduction so long as the original work is given appropriate credit. Contents may not be used for commercial purposes, or adapted, remixed, transformed or built upon. (https://creativecommons.org/ licenses/by-nc-nd/4.0/)

Thieme Medical and Scientific Publishers Pvt. Ltd., A-12, 2nd Floor, Sector 2, Noida-201301 UP, India
} 


\section{Introduction}

Over the last decades, there has been a tremendous progress in the composition of orthodontic wires from stainless steel to Nickel-Titanium. ${ }^{1}$ Nickel-Titanium wires have great mechanical properties such as superelasticity, thermal shape memory, good corrosion resistance, and good biocompatibility that help orthodontists solve malocclusions easily. ${ }^{2}$ The Titanium oxide layer covering the surface of NickelTitanium orthodontic wires during galvanic corrosion is responsible for its biocompatibility in the oral cavity ${ }^{3}$ and this layer determine the corrosion resistance of the wire.

Bacterial adhesion to orthodontic wires leads to a biodegradation of the wire's surface and might lead to a decrease in corrosion resistance. ${ }^{4}$ Streptococcus mutans is present in great amount in the plaques of orthodontic patients with fixed appliances ${ }^{5}$ because fixed orthodontic appliances have an effect on the oral microbiota. ${ }^{6}$ Acids produced by S. mutans, cause enamel demineralization. ${ }^{7,8}$ Therefore, S. mutans adhesion to orthodontic materials could be regarded as a key factor in the pathogenesis of enamel demineralization during orthodontic treatment. ${ }^{7}$

Because white spot lesions are the major complications of a fixed orthodontic treatment, patients must undertake a good oral hygiene regimen. All orthodontists recommend to their patients a daily use of fluoridated toothpastes and mouthwashes because fluoride is responsible for the formation of calcium fluoride complex that adheres to the teeth and leads to its remineralization protecting enamel against acid attack. ${ }^{1}$ Nevertheless, it has been widely proven that the increase in the concentration of fluoride leads to a decrease in the corrosion resistance of Nickel-Titanium wires. ${ }^{9}$ The corrosion amount of Nickel-Titanium alloy in the presence of fluoride ions increases up to 1,000 times and a decrease in the passive film breakdown potential is also observed. ${ }^{10}$

The incidence of enamel demineralization after fixed orthodontic treatment can involve up to $50 \%$ of patients. The incidence of such white spot lesions around orthodontic brackets has been demonstrated within 1 month of the treatment. ${ }^{11,12}$ Thus, an anti-bacterial and anti-adherent agent is of a major concern to limit the white spot lesion occurrence during orthodontic treatment.

Several antimicrobial agents have been described in the literature, among them Syzygium aromaticum essential oil, which mainly constitute Eugenol. ${ }^{13}$ Eugenol (4-allyl-2methoxyphenol) is an aromatic molecule found in essential oils and hydrosols of a wild range of plants. ${ }^{14}$ Several researchers have reported that this molecule has antibacterial, antiviral, antioxidant, anti-inflammatory and analgesic effects. ${ }^{15,16}$ Furthermore, Eugenol is widely used in dentistry to treat toothache and pulpitis ${ }^{17}$ but its anti-adherent effect on orthodontic wires has never been studied.

It has also been reported that Eugenol has an anticorrosive effect against Nickel-Titanium orthodontic wires. ${ }^{18}$ Because adding fluoride to hygiene regimen of orthodontic patients wearing fixed orthodontic appliance have a negative effect on corrosion resistance of wires, Eugenol may constitute an alternative.
The purpose of this study was to compare the antibacterial effect of two types of Eugenol against $S$. mutans and then assess the effect of different concentrations of two types of Eugenol on S. mutans adhesion on NiTi orthodontic wires in comparison to in vivo wires with fluoride-based hygiene regimen.

\section{Materials and Methods}

\section{Orthodontic Wires}

This study was done in two parts, the first was conducted in vitro on as received Nickel-Titanium orthodontic wires and the second was done on retrieved wires from orthodontic patients with in vivo conditions.

NiTi wire with equiatomic composition ( $\mathrm{NiTi}, 3 \mathrm{M}$ ) was used for the in vitro study. Samples were cut into segments of $20 \mathrm{~mm}$ from the straight part of as received wires. Before testing, wires were rinsed thrice in water and immersed in acetone for 5 minutes.

Regarding retrieved wires, three dimensions of NiTi orthodontic wires were selected representing different stages of the orthodontic treatment: $0.016,0.016 \times 0.022$, and $0.017 \times 0.025$ inch. Patients were given hygiene instructions and they brushed their teeth thrice a day with their usual toothpaste containing fluoride (1,240 ppm). After one month, arch wires were retrieved cautiously with a weingart plier to avoid any contact with oral mucosa and were stored separately in phosphate buffered solution (PBS) in a $50 \mathrm{~mL}$ of sterile falcon tube until further use. They were conducted immediately to the laboratory and in sterile conditions $20 \mathrm{~mm}$ from the straight part of each wire were cut.

\section{Bacterial Culture}

Saliva was obtained from adult patients with caries. Samples were collected in sterile recipients and were immediately transferred to the laboratory. They were diluted at 1/100 in sterile physiologic serum. Next, $50 \mu \mathrm{L}$ of aliquots were spread in Mitis Salivarius Agar (MSA). After 48 hours of anaerobic incubation at $37^{\circ} \mathrm{C}$, the isolated bacteria were transferred and spread in another MSA medium and incubated for 24 hours. After 2 weeks of serial cultures, the bacteria isolated were identified using Rapid ID 32 strep galleries biochemical and enzymatic tests (BioMerieux, SA). The bacteria were added to Ringer solution enriched with tryptic soy broth (TSB) medium to obtain active cultures.

\section{Growth Inhibition Produced by Well-Diffusion Method} Streptococcus mutans was inoculated in the brain heart infusion (BHI) broth and incubated for 24 hours, to the point when growth was considered to be in the logarithmic phase. The density of the bacterial suspension was adjusted with sterile PBS to match the density of McFarland's standard 0.5. The bacterial broth suspension was streaked evenly onto the BHI agar plates with a cotton swab.

After the inoculum had dried, 8-mm single wells were made using a cork borer. Two types of Eugenol were studied, the biological Eugenol made by hydrodistillation of S. aromaticum and the Eugenol sold by manufacturers and used in dentistry 


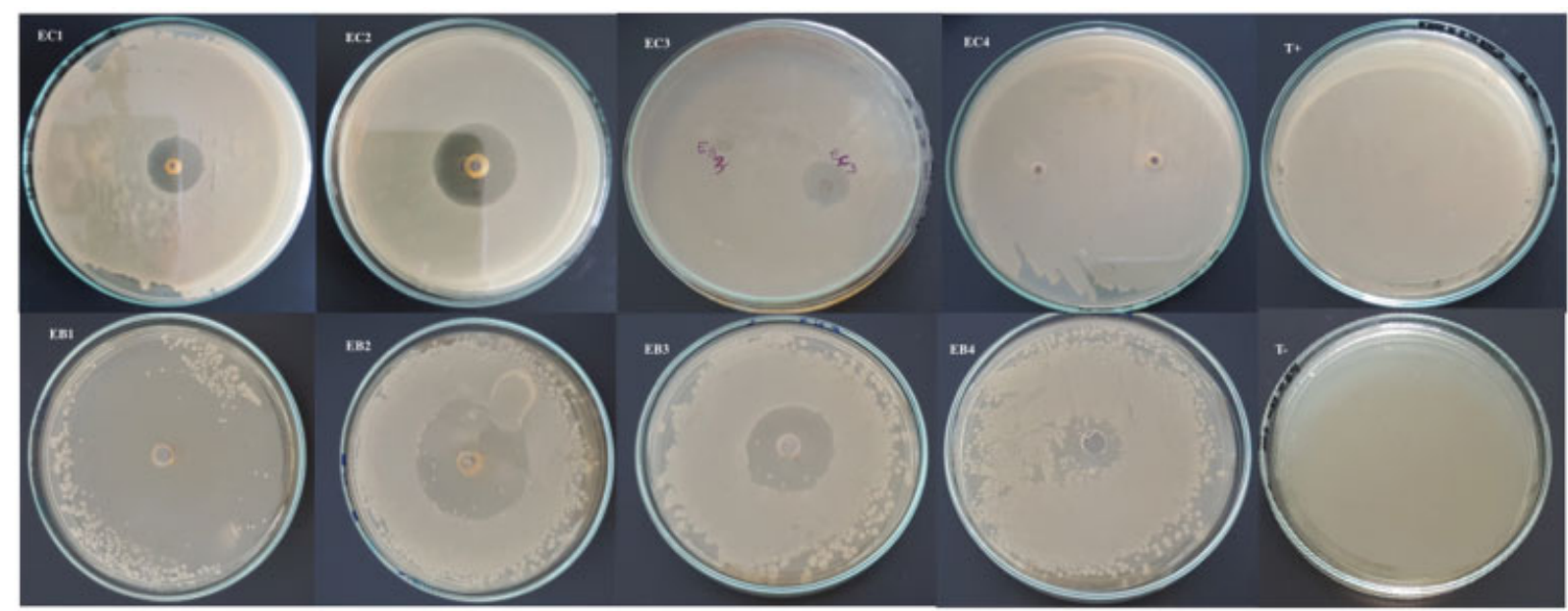

Fig. 1 Growth inhibition of eugenol against Streptococcus mutans produced by well-diffusion method. EC1, EC2, EC3, and EC4 correspond to chemical Eugenol with $100 \%, 50 \%, 25 \%$, and $12.5 \%$, respectively. EB1, EB2, EB3, and EB4 correspond to biological Eugenol with 100\%, 50\%, 25\%, and $12.5 \%$, respectively. $\mathrm{T}-$ : negative control. $\mathrm{T}+$ : positive control.

to prepare zinc oxide eugenol paste. The two types of Eugenol were diluted to four concentrations: $100 \%, 50 \%, 25 \%$, and $12.5 \%$. Then, $50 \mu \mathrm{L}$ of each concentration of the two types of Eugenol was added and the plates were kept incubated at $37^{\circ} \mathrm{C}$ for 24 hours. After incubation, diameters of the growth inhibition zones were measured in $\mathrm{mm}$ at three different points in the circle zones.

\section{Assessment of the Bacterial Adhesion of Streptococcus mutans on Wire's Surface}

Artificial saliva (Ringer solution) was used as base and as the previous study, the same types of Eugenol and the same dilutions were used in the anti-adherent study. Dilutions were prepared using sterile distilled water and dimethyl sulfoxide (DMSO) was used to solubilize Eugenol.

Each wire (in vitro and in vivo retrieved wires) was cultured separately to avoid cross contamination in a tube containing $5 \mathrm{~mL}$ of artificial saliva with $50 \mu \mathrm{L}$ of bacterial suspension to obtain a $1 / 100$ concentration. The different concentrations of the two types of Eugenol were then added and all tubes were incubated at $37^{\circ} \mathrm{C}$ with agitation during 48 hours.

After incubation, wires were removed from the solutions to assess the adhesion of $S$. mutans. Each wire was rinsed thrice with a saline solution and transferred to a new microtube containing $1 \mathrm{~mL}$ of sterile distilled water. Then, microtubes were sonicated to detach adherent bacteria using four pulses of 30 seconds with intermittent cooling as described by Yang et al. ${ }^{19}$ Serial dilution with sterile distilled water was then done for each microtube. Next, $100 \mathrm{~mL}$ of the two dilutions $10^{-3}$ and $10^{-5}$ after vortex were seeded in the BHI single agar plate. Positive (with wires and bacteria and without Eugenol) and negative (wires without bacteria) controls were used for each dilution. Petri dishes were then incubated at $37^{\circ} \mathrm{C}$ during 24 hours. Each group consists of three samples.

Colony counting was done by colony forming units to unitary surface $(\mathrm{CFU} / \mathrm{mL})$.

\section{SEM Analysis}

A SEM analysis was done to each wire to observe whether bacteria have been removed from the wire surface or not. Images were taken at magnification $\times 250$ and $\times 2,500$.

\section{Statistical Analysis}

The collected data were analyzed with the RStudio software. Results were presented in means and standard deviations. Because the test for normality showed that the data were normal to find the significant differences between the simples. ANOVA test was performed along with post-hoc $t$-test and Bonferroni comparison were used to elucidate statistical differences.

\section{Results}

Inhibitory Effect of Eugenol on Streptococcus mutans

-Fig. 1 shows diameters of inhibition zones in the presence of each dilution of the two types of Eugenol. The width of halos increases by increasing the concentration of Eugenol, $42 \mathrm{~mm}$ with $100 \%$ of biological Eugenol and $16 \mathrm{~mm}$ with $12.5 \%$ of the same Eugenol. Halos are bigger in biological Eugenol (42 $\mathrm{mm}$ at $100 \%)$ than in chemical Eugenol $(23 \mathrm{~mm}$ at $100 \%)$.

-Table 1 shows the analysis of the inhibitory effect regarding biological Eugenol that has a more inhibitory effect on S. mutans than the chemical one in all concentrations. For the two types of Eugenol, decreasing the concentration of the

Table 1 Zone of inhibition of Streptococcus mutans following different concentrations of the two types of Eugenol (mm)

\begin{tabular}{|l|l|l|}
\hline & Biological Eugenol & Chemical Eugenol \\
\hline $100 \%$ & $42 \mathrm{~mm}$ & $23 \mathrm{~mm}$ \\
\hline $50 \%$ & $41 \mathrm{~mm}$ & $22 \mathrm{~mm}$ \\
\hline $25 \%$ & $24 \mathrm{~mm}$ & $19 \mathrm{~mm}$ \\
\hline $12.5 \%$ & $16 \mathrm{~mm}$ & $0 \mathrm{~mm}$ \\
\hline
\end{tabular}


Table 2 Mean and standard deviation of colony forming units of Streptoccocus mutans in the presence of the different concentrations of biological and chemical Eugenol in vitro

\begin{tabular}{|l|l|l|l|l|}
\hline & \multicolumn{2}{|l|}{ Biological Eugenol } & \multicolumn{2}{l|}{ Chemical Eugenol } \\
\hline & $\begin{array}{l}\text { Mean }\left(\times 10^{5}\right) \\
(\mathrm{CFU} / \mathrm{mL})\end{array}$ & SD & $\begin{array}{l}\text { Mean }\left(\times 10^{5}\right) \\
(\mathrm{CFU} / \mathrm{mL})\end{array}$ & SD \\
\hline $100 \%$ & $0.2^{\mathrm{ABC} * *}$ & 0.035 & $0.25^{\mathrm{HJ} * *}$ & 0.212 \\
\hline $50 \%$ & $5.25^{\mathrm{D} *}$ & 1.06 & $10^{\mathrm{K} *}$ & 0.989 \\
\hline $25 \%$ & $10.1^{\mathrm{BF} *}$ & 0.424 & $14.5^{\mathrm{L} *}$ & 5.939 \\
\hline $12.5 \%$ & $15.15^{\mathrm{ADEG} * * *}$ & 1.626 & $30.35^{\mathrm{H} * *}$ & 6.434 \\
\hline Positive control & $37.65^{\mathrm{CEFG} * * *} \mathrm{JKL}$ & \pm 3.747 & \\
\hline Negative control & 0 & & 0 & \\
\hline F value & 117.1 & 25.3 & \\
\hline
\end{tabular}

Note: Within a column, values with the same capital superscript letter indicate statistically significant difference based on the Bonferroni multiple comparison tests at $\alpha=0.05$.

No statistically significant difference was present between the positive control and the chemical Eugenol at $12.5 \%$.

${ }^{*} p<0.05$.

${ }^{* *} p<0.01$.

${ }^{* * *} p<0.001$

molecule decreases the zone of inhibition of S. mutans. At concentration of $12.5 \%$, the chemical Eugenol has no antibacterial effect on $S$. mutans. In the absence of Eugenol, a bacterial growth has been noted and no halo has been detected $(0 \mathrm{~mm})$.

\section{Colony Forming Units Regarding the Different Concentrations of the Two Origins of Eugenol}

- Table 2 shows that biological Eugenol has a more antiadherent effect than the chemical one. The more the concentration of the two types of Eugenol decreases, the more the anti-adherent effect decreases too.

\section{Wires with In Vivo Conditions}

The mean of colony forming units regarding in vivo wires is higher than the one in the presence of Eugenol. The third wire with the $0.017 \times 0.025$ size shows the higher amount of adherent bacteria (-Table $\mathbf{3}$ ).

\section{SEM Wires Surface Analysis}

-Fig. 2 shows SEM images of NiTi wires in the presence of $S$. mutans after immersion in different concentrations of the two types of Eugenol. For the chemical Eugenol, the $100 \%$ concentration (EC1) shows an image with scratches and black depositions on the surface of wire while in biological Eugenol (EB1), and at the same concentration, the surface of

Table 3 Mean and standard deviation of colony forming units of bacteria from wires with in vivo conditions

\begin{tabular}{|l|l|l|}
\hline & $\begin{array}{l}\text { Mean }\left(\times \mathbf{1 0}^{\mathbf{5}}\right) \\
(\mathrm{CFU} / \mathrm{mL})\end{array}$ & SD \\
\hline Wire 1 & 33.05 & 4.03 \\
\hline Wire 2 & 39.7 & 6.646 \\
\hline Wire 3 & 50.5 & 5.656 \\
\hline
\end{tabular}

the wire is similar to the control with deposition of white crystals on its surface. In both wires, there are no bacteria in the surface.

With $50 \%$ of Eugenol concentration, NiTi wire with chemical Eugenol shows white irregular surfaces all over the wire (EC2) and the biological Eugenol has a surface similar to the control with some pitting in the surface of the wire (EB2).

With 25\% of Eugenol concentration, NiTi wires with chemical Eugenol demonstrate a surface with white appositions (EC3) while with biological Eugenol, there is a large white surface on the wire (EB3).

With $12.5 \%$, white crystals have been observed on the surface of the wire with chemical Eugenol and (EC4) with biological Eugenol, there are black points on the surface of the wire (EB4).

With in vivo wire $\mathrm{N}^{\circ} 1$, there is a lot of pitting and deep scratches on the surface of the wire. In the in vivo wire $\mathrm{N}^{\circ} 2$ and 3, cocci and bacilli bacteria have been observed on the surface both wires forming complex units.

\section{Discussion}

The aim of this study was to compare the antibacterial effect of two types of Eugenol (a Biological one made by hydrodistilation of $S$. aromaticum and the commercial one used in dentistry) against S. mutans. Results of this study confirm that Eugenol has an anti-bacterial effect on $S$. mutans. Biological Eugenol has more antibacterial effects than the one sold in commerce. Xu (2013) reported in his study that Eugenol demonstrated significant inhibitory effects against acid production by $S$. mutans. The synthesis of water-insoluble glucans by glucosyltransferases was reduced by eugenol. ${ }^{20}$ At $12.5 \%$ of chemical Eugenol, there is no antibacterial effect on $S$. mutans while with the biological Eugenol, an inhibition zone of $16 \mathrm{~mm}$ was observed. This result confirms that the biological Eugenol is more efficient on S. mutans ++ . 


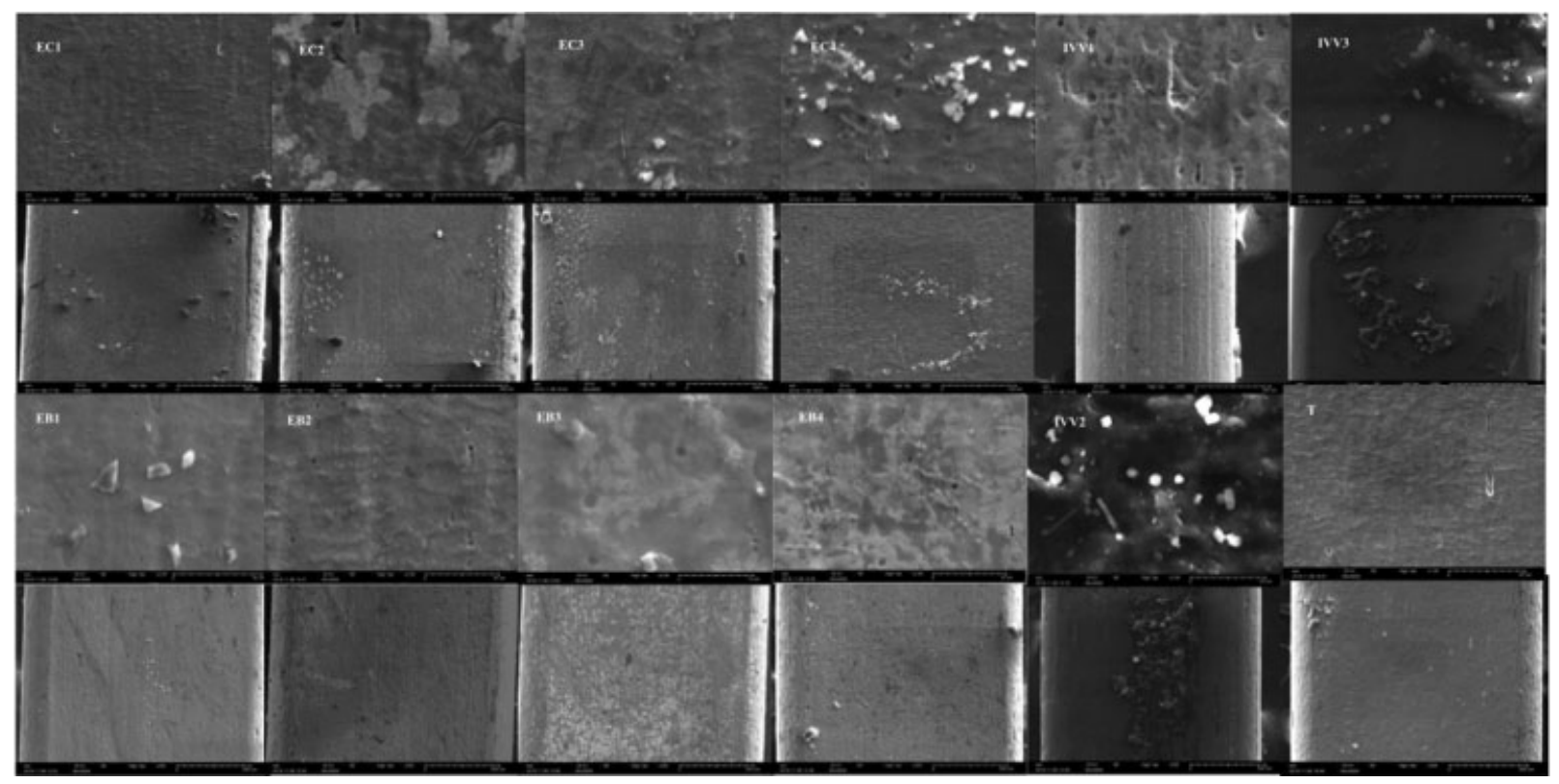

Fig. 2 SEM analysis of NiTi wires in different solutions at the end of the experiment. EC1, EC2, EC3, and EC4 correspond to chemical Eugenol with $100 \%, 50 \%, 25 \%$, and $12.5 \%$, respectively. EB1, EB2, EB3, and EB4 correspond to biological Eugenol with $100 \%, 50 \%, 25 \%$, and $12.5 \%$, respectively. IVV1, IVV2, and IVV3 represent wires with in vivo conditions at 016 inch, $0.016 \times 0.022$ inch, and $0.017 \times 0.025$ inch, respectively. $\mathrm{T}$ : control. Each wire is represented by two images one on the top with $\times 2,500$ magnification and other on the bottom with $\times 250$ magnification. White surfaces and crystals correspond to appositions on the surface of the wire indicating a corrosion inhibition, and black pitting matches with the degradation of the wire surface. IVV2 and IVV3 show the amount of adherent bacteria on their surfaces.

Several aromatic plants has been used in dentistry for their beneficial effects, they are an interesting source of ecofriendly, inexpensive, and available new large spectrum antimicrobials with low levels of cutaneous cytotoxicity and environmental toxicity. ${ }^{21-23}$ Besides, because sodium fluoride added to toothpastes increases the corrosion process of Nickel-Titanium orthodontic wires, providing a prosperous environment for bacterial colonization, it might be interesting to search for another alternative that can have anti-bacterial and anti-corrosive effects at the same time.

Hydrodistillation of S. aromaticum yield clove essential oil, which is an aromatic and volatile substance. It has multiple properties such as antimicrobial, antioxidant, and anti-inflammatory effects that make it widely used in different areas. The US Food and Drug Administration listed it like "generally regarded as safe" chemical. ${ }^{24}$ The main constituent of this essential oil is Eugenol (4-allyl-2-methoxyphenol). It has a large spectrum antimicrobial ability against several bacteria species. Eugenol displays also an essential activity against anaerobic oral bacteria like S. mutans. ${ }^{25,26}$ Other essential oils have been described and showed an antibacterial effect. Among them, Origanum dubium oil and Cinnamomum cassia oil with a mean zone of inhibition of $31 \mathrm{~mm}$ and $38.67 \mathrm{~mm}$, respectively, ${ }^{27}$ which are minor than the results found with Eugenol in this study ( $42 \mathrm{~mm}$ ), suggesting that the biological eugenol is more effective on S. mutans. The second purpose of this study was to evaluate the effect of different concentrations of these two types of Eugenol on S. mutans adhesion on NiTi orthodontic wires. Results show that biological Eugenol has more anti-adherent effects than the chemical one and increasing the concentration of Eugenol leads to an increase in the anti-adherent effect on S. mutans. The difference in results between the two types of Eugenol might be due to the mechanism of preparation of the solution and the molecule purification.

In fact, white spot lesions (WSL) are the most common side effects of a fixed orthodontic treatment. Studies have demonstrated that the amount of S. mutans in biofilm over WSL is high. ${ }^{28}$ Plaque accumulation around orthodontic brackets often results in enamel white spot formation adjacent to the brackets. This plaque is composed of various microorganisms of which $S$. mutans is the most cariogenic. Its adherence to the fixed appliance is largely contributed to the bracket material. S. mutans along with glycosyltransferase degrades sucrose to make insoluble glucans that also attach to the tooth surface, providing ideal sites for oral bacteria to inhabit. The resulting complex of glucan and various bacteria then creates an oral biofilm that is the mature stage of dental plaque. As plaque accumulates, acidic compounds such as fructose and other fatty acids degrade the enamel surface of the teeth through a process known as dental caries. ${ }^{29}$

Mechanical removal of biofilm by brushing is very effective. This is why orthodontists prescribe fluoridated toothpastes and mouthwashes to help patients controlling plaque formation. ${ }^{30,31}$ Nevertheless, as reported in this study, the highest amount of adherent bacteria was observed with wires in vivo among patients who brushed their teeth with fluoridated toothpaste. This suggests that Eugenol might be considered as alternative to fluoride. It might constitute an adjuvant element to hygiene regimen because it has an 
antibacterial effect along with anti-adherent effect against $S$. mutans. Eugenol can also be used in toothpastes and mouthwashes for patients wearing fixed appliances from the beginning of their treatment. Further studies have to be done to determine the minimum and the maximum dose to avoid the side effects of Eugenol.

Other substances described in the literature such as Rhus coriaria L. water extract had significant antibacterial properties against $S$. mutans and is able to inhibit bacterial biofilm formation on orthodontic wires. ${ }^{32}$ Furthermore, Dias has confirmed in her study that $S$. mutans biofilms adhesion to brackets were susceptible to $\mathrm{CHX}$ treatment.

\section{Conclusions}

This study confirmed that Eugenol has an antibacterial effect against $S$. mutans. The biological Eugenol is more effective than the one sold in commerce. Furthermore, Eugenol obtained in laboratory by hydrodistillation of S. aromaticum has more anti-adherent activity against $S$. mutans on NickelTitanium orthodontic wires than the Eugenol used in dentistry and found in commerce. Orthodontists should be aware of $S$. mutans adhesion because it causes white spot lesions, especially when using the same arch wire for a long period of time.

\section{Conflict of Interest}

None declared.

\section{Acknowledgments}

We want to thank Pr. Mohammed Berrada and Said Mansouri for their help in the SEM analysis and the PhD students Derdak Reda and Sakoui Soraya for their help and contribution to this study.

\section{References}

1 Mirjalili M, Momeni M, Ebrahimi N, Moayed MH. Comparative study on corrosion behaviour of Nitinol and stainless steel orthodontic wires in simulated saliva solution in presence of fluoride ions. Mater Sci Eng C 2013;33(04):2084-2093

2 Proffit WR, Fields HW, Sarver DM. Contemporary Orthodontics. 5th ed. Mosby Elsevier; 2007

3 Shabalovskaya SA. On the nature of the biocompatibility and on medical applications of NiTi shape memory and superelastic alloys. Biomed Mater Eng 1996;6(04):267-289

4 Mei L, Busscher HJ, van der Mei HC, Ren Y. Influence of surface roughness on streptococcal adhesion forces to composite resins. Dent Mater 2011;27(08):770-778

5 Lee H-J, Park H-S, Kim K-H, Kwon T-Y, Hong S-H. Effect of garlic on bacterial biofilm formation on orthodontic wire. Angle Orthod 2011;81(05):895-900

6 Lucchese A, Bondemark L, Marcolina M, Manuelli M. Changes in oral microbiota due to orthodontic appliances: a systematic review. J Oral Microbiol 2018;10(01):1476645

7 Lee SP, Lee SJ, Lim BS, Ahn SJ. Surface characteristics of orthodontic materials and their effects on adhesion of mutans streptococci. Angle Orthod 2009;79(02):353-360

8 Kwon TY, Hong SH, Kim YK, Kim KH. Antibacterial effects of 4-META/MMA-TBB resin containing chlorhexidine. J Biomed Mater Res B Appl Biomater 2010;92(02):561-567
9 Heymann GC, Grauer D. A contemporary review of white spot lesions in orthodontics. J Esthet Restor Dent 2013;25(02):85-95

10 Lee T-H, Wang C-C, Huang T-K, Chen L-K, Chou M-Y, Huang H-H. Corrosion resistance of titanium-containing dental orthodontic wires in fluoride-containing artificial saliva. Alloys Compd. 2009; 488:482-489

11 Gorelick L, Geiger AM, Gwinnett AJ. Incidence of white spot formation after bonding and banding. Am J Orthod 1982;81 (02):93-98

12 Artun J, Brobakken BO. Prevalence of carious white spots after orthodontic treatment with multibonded appliances. Eur J Orthod 1986;8(04):229-234

13 Mandava K, Batchu UR, Kakulavaram S, et al. Design and study of anticaries effect of different medicinal plants against S.mutans glucosyltransferase. BMC Complement Altern Med 2019;19(01): 197

14 Walsh SE, Maillard JY, Russell AD, Catrenich CE, Charbonneau DL, Bartolo RG. Activity and mechanisms of action of selected biocidal agents on Gram-positive and -negative bacteria. J Appl Microbiol 2003;94(02):240-247

15 Chami N, Bennis S, Chami F, Aboussekhra A, Remmal A. Study of anticandidal activity of carvacrol and eugenol in vitro and in vivo. Oral Microbiol Immunol 2005;20(02):106-111

16 Yeon KY, Chung G, Kim YH, et al. Eugenol reverses mechanical allodynia after peripheral nerve injury by inhibiting hyperpolarization-activated cyclic nucleotide-gated (HCN) channels. Pain 2011;152(09):2108-2116

17 Frisch J, Bhaskar SN. Tissue response to eugenol-containing periodontal dressings. J Periodontol 1967;38(05):402-408

18 Fatene N, et al. Assessement of the electrochemical behavior of Nickel-Titanium based orthodontic wires: effect of different solutions in comparison to fluoride. J Clin Exp Dent 2019;11 (05): e414-e420

19 Yang IH, Lim BS, Park JR, Hyun JY, Ahn SJ. Effect of orthodontic bonding steps on the initial adhesion of mutans streptococci in the presence of saliva. Angle Orthod 2011;81(02):326-333

$20 \mathrm{Xu}$ J-S, Li Y, Cao X, Cui Y. The effect of eugenol on the cariogenic properties of Streptococcus mutans and dental caries development in rats. Exp Ther Med 2013;5(06):1667-1670

21 Yasin P, Wanachantararuk P, Jidaphatinoi J, Dumri K. Antimicrobial activity of some Thai aromatic plants against oral pathogens including halitosis. Asian J Pharm Clin Res 2019;12(01):465-468

22 Sofrata A, Santangelo EM, Azeem M, Borg-Karlson AK, Gustafsson A, Pütsep K. Benzyl isothiocyanate, a major component from the roots of Salvadora persica is highly active against Gram-negative bacteria. PLoS One 2011;6(08):e23045

23 Sinha DJ, Sinha AA. Natural medicaments in dentistry. Ayu 2014; 35(02):113-118

24 Amorati R, Foti MC, Valgimigli L. Antioxidant activity of essential oils. J Agric Food Chem 2013;61(46):10835-10847

25 Jafri H, Banerjee G, Khan MSA, Ahmad I, Abulreesh HH, Althubiani AS. Synergistic interaction of eugenol and antimicrobial drugs in eradication of single and mixed biofilms of Candida albicans and Streptococcus mutans. AMB Express 2020;10(01):185

26 Xu J-G, Liu T, Hu Q-P, Cao X-M. Chemical composition, antibacterial properties and mechanism of action of essential oil from clove buds against Staphylococcus aureus. Molecules 2016;21(09):1194

27 Karadağlıoğlu Öİ, Ulusoy N, Bașer KHC, Hanoğlu A, Şık İ Antibacterial activities of herbal toothpastes combined with essential oils against Streptococcus mutans. Pathogens 2019;8(01):20

28 Gross EL, Leys EJ, Gasparovich SR, et al. Bacterial 16S sequence analysis of severe caries in young permanent teeth. J Clin Microbiol 2010;48(11):4121-4128

29 Rammohan SN, Juvvadi SR, Gandikota CS, Challa P, Manne R, Mathur A. Adherence of Streptococcus mutans and Candida albicans to different bracket materials. J Pharm Bioallied Sci 2012;4 (Suppl 2):S212-S216 
30 Vermaire JH, van Loveren C, Brouwer WB, Krol M. Value for money: economic evaluation of two different caries prevention programmes compared with standard care in a randomized controlled trial. Caries Res 2014;48(03):244-253

31 Costa MR, Silva VC, Miqui MN, Sakima T, Spolidorio DM, Cirelli JA. Efficacy of ultrasonic, electric and manual toothbrushes in patients with fixed orthodontic appliances. Angle Orthod 2007; 77(02):361-366

32 Vahid-Dastjerdi E, Sarmast Z, Abdolazimi Z, Mahboubi A, Amdjadi P, Kamalinejad M. Effect of Rhus coriaria L. water extract on five common oral bacteria and bacterial biofilm formation on orthodontic wire. Iran J Microbiol 2014;6(04):269-275 